\title{
Publication dynamics in gastroenterology and hepatology over the last decade in Greece: a SCImago-based study
}

\author{
Stamatia C. Vorri, Aikaterini Karagouni, Stefanos Karamaroudis, Panagiota Katsouli, Aliki Stamou, \\ George D. Dimitriadis, Konstantinos Triantafyllou \\ Medical School, National and Kapodistrian University of Athens, "Attikon" University General Hospital, Athens, \\ Greece
}

\begin{abstract}
Background There is evidence that the financial crisis has deleteriously affected scientific output. We aimed to assess the dynamics of Greek publications in gastroenterology and hepatology over the last ten years.

Methods Data were collected from SCImago. The average annual growth rate (AAGR) of total and citable documents published in Greece in the field of gastroenterology and hepatology was compared with that of cardiology, surgery, and radiology. In addition, it was compared with the corresponding rates in Belgium, Ireland and Portugal.

Results The annual number of Greek publications (total and citable documents) remained relatively unchanged in the field of gastroenterology and hepatology from 2006-2011. During the following years, we detected a negative AAGR, with values of $-12 \%,-3.3 \%,-9.5 \%$ and $-5.4 \%$ for gastroenterology-hepatology, cardiology, surgery and radiology, respectively. During the same period, the AAGR of the citable documents of the respective specialties was $-8.6 \%,-3.7 \%$, $-9.8 \%$, and $-5.1 \%$. Comparison of the 4 European countries in the field of gastroenterology and hepatology revealed that publications from Portugal rose massively (AAGR $+24.6 \%$ and $+20.6 \%$ for total and citable documents, respectively), almost reaching the number of Greek publications in 2015. The number of publications from Belgium and Ireland remained essentially unchanged after 2011 , with AAGR values of $-0.7 \%$, and $-2.1 \%$ for total and $-1.1 \%$ and $-1.4 \%$ for citable documents, respectively.

Conclusion The publication output in the field of gastroenterology and hepatology decreased significantly after the outbreak of the financial crisis in Greece, not only in relation to other medical specialties, but also compared to the output of other European countries with or without fiscal austerity measures.
\end{abstract}

Keywords Gastroenterology and hepatology, publications, SCImago, bibliometric, financial crisis Ann Gastroenterol 2018; 31 (1): 1-4

Second Department of Internal Medicine, Research Institute and Diabetes Center, Medical School, National and Kapodistrian University of Athens, "Attikon" University General Hospital, Athens, Greece

Conflict of Interest: None

Correspondence to: Konstantinos Triantafyllou, Associate Professor of Gastroenterology, Hepatogastroenterology Unit, Second Department of Internal Medicine - Propedeutic, Research Institute and Diabetes Center, Medical School, National and Kapodistrian University, "Attikon" University General Hospital, 1, Rimini Street, 12462 Athens, Greece, e-mail: ktriant@med.uoa.gr

Received 28 June 2017; accepted 10 October 2017; published online 16 November 2017

DOI: https://doi.org/10.20524/aog.2017.0212

\section{Introduction}

Bibliometric studies have been conducted over the last years to assess the scientific productivity of different world regions in several scientific fields. Focusing on biomedical sciences, investigators use their own distinctive methodologies and choose the geographic region and scientific field to investigate more or less arbitrarily. Some of them compare geographic areas worldwide by analyzing global data in several nonspecific fields, while others assess the productivity in a specific field. Data for these investigations derive from several databases, each of which has pros and cons: PubMed, Web of Science, Scopus, Google Scholar and SCImago are among them. 
There is strong evidence that austerity measures, implemented during the financial crisis that emerged in Europe in 2009, have had a deleterious effect on the scientific output in the region; however, this effect is not uniform among countries $[1,2]$. Our study aimed to quantitatively evaluate the publication productivity of Greek researchers in the field of gastroenterology and hepatology during the decade 2006-2015 and to assess any temporal effect of the financial crisis on it.

\section{Materials and methods}

We retrieved data from the SCImago website [3]. SCImago is an online platform that ranks journals and countries in several knowledge fields based on bibliometric markers, such as total documents per year and over a 3-year period, total references, citable documents, cites per document, references per document and country, the H-index and the SCImago Journal Rank indicator (SJR). The ranking is made annually using Elsevier's subscription-based Scopus database, which includes a remarkably wide range and large number of scientific journals, not only in English but in 50 other languages. The SCImago webpage is updated daily, it offers open access and is simple to use, thereby providing sophisticated tools for data visualization. Also, according to some researchers, the SJR indicator is the best alternative to the commonly used "impact factor", given its ability to exclude self-citations and to take into account not only the number of citations of a publication, but also their quality, depending on the "prestige" of the citing journal [4].

We recorded the number of total documents and the number of citable (original articles, reviews and conference papers) documents in the field of gastroenterology and hepatology (summed data) published during the decade 20062015. We had two comparators: 1 ) the productivity (total and citable documents) in 3 other medical specialties in Greece, namely cardiology (cardiology and cardiovascular medicine documents in SCImago), surgery, and radiology (including radiology, nuclear medicine and imaging documents in SCImago); and 2) the scientific output in terms of total and citable documents in gastroenterology and hepatology in 3 other European Union (EU) countries, namely Belgium, Portugal and Ireland. The former two countries' population size is similar to that of Greece, according to the United Nations' 2015 revision of World Population Prospects [5], while Ireland, Portugal and Greece were subjected to austerity measures after the emergence of the financial crisis in the EU.

Data retrieved from the SCImago website were used to calculate the annual growth rate (the value at the end minus the value at the start of each year, divided by the starting value) and the average annual growth rate (AAGR), using the following formula:

$\mathrm{AAGR}=($ growth rate in period $\mathrm{A}+$ growth rate in period $\mathrm{B}+$ growth rate in period $\mathrm{C}+\ldots+$ growth rate in period $\mathrm{X}) \div$ number of periods

Data are presented in a descriptive manner and with figures produced using the OpenOffice Calc computer software.

No Ethics Committee approval was required for this study.

\section{Results}

During the period 2006-2011, the annual number of Greek publications (total and citable documents) remained relatively unchanged in the field of gastroenterology and hepatology. A similar pattern was also observed for radiology, while there was an increase in the numbers of total documents per year (albeit with different slopes) in the fields of cardiology and surgery (Fig. 1).

Subsequently, there was a decrease in the number of total documents in gastroenterology and hepatology, from 354 in 2011 to 212 in 2015, representing an AAGR of $-12 \%$. Over the same period, the AAGR was $-3.3 \%,-9.5 \%$ and $-5.4 \%$ for cardiology, surgery and radiology, respectively.

Similarly to the total documents, the performance of the 4 medical specialties regarding citable documents remained invariable during the first half of the observation period. However, a decrease in the output of citable documents was observed after 2011, with AAGRs of $-8.6 \%,-3.7 \%,-9.8 \%$, and $-5.1 \%$ for the 4 specialties, respectively. However, in the last two years cardiology showed signs of recovery, with an AAGR of $4 \%$ (Fig. 2).

Figs. 3 and 4 illustrate the comparison of the 4 EU countries regarding the number of total and citable documents in the field of gastroenterology and hepatology over the last decade. Regarding total documents, in 2011 Greece ranked first $(n=354)$, followed by Belgium $(n=331)$, Ireland $(n=138)$ and Portugal $(n=86)$. Subsequently, in contrast to the negative AAGR of the Greek publications after $2011(-12 \%)$, the number of total documents from Belgium and Ireland remained essentially invariable, with AAGRs of $-0.7 \%$ and $-1.1 \%$, respectively. In contrast, Portugal achieved a great increase in publication numbers with an AAGR of $24.6 \%$ for total documents after 2011 . This huge increase, combined with the decrease in the Greek publications, resulted in almost an equalization of the two countries' total documents in 2015 (212 for Greece vs. 201 for Portugal), as shown in Fig. 3.

The pattern of the $4 \mathrm{EU}$ countries regarding the number of citable documents was similar, as shown in Fig. 4. At the end of the studied period, Belgium $(n=270)$ replaced Greece $(n=181)$ and Portugal $(n=132)$ replaced Ireland $(n=93)$ in the first and third places of the ranking, compared to 2006. This finding can be mainly attributed to the differences in AAGR between the countries after 2011: $-8.6 \%$ for Greece vs. $2.1 \%$ for Belgium and $-1.4 \%$ for Ireland vs. $20.6 \%$ for Portugal.

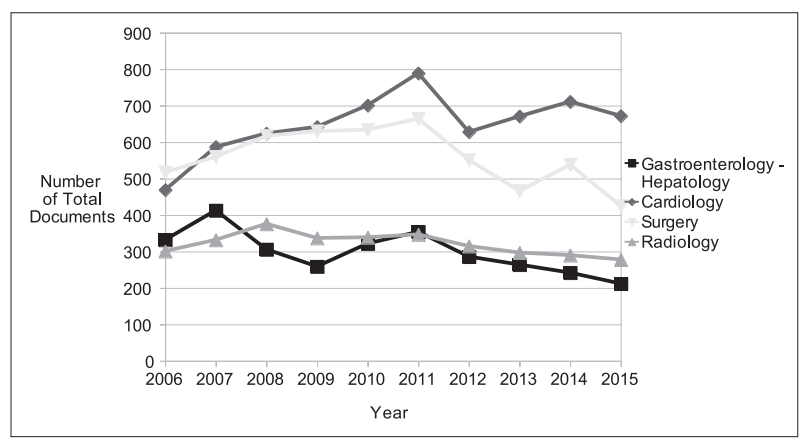

Figure 1 SCImago numbers of total documents in the fields of gastroenterology-hepatology, cardiology, surgery and radiology in Greece during the decade 2006-2015 


\section{Discussion}

Our observations indicate that, over the last decade, the Greek publication output in the field of gastroenterology and hepatology has been through major agitation, with a significant decrease not only in the number of scientific papers, but also in the rate at which they are being published. Although this downward trend was observed in all 4 of the Greek medical fields examined, it was most prominent in gastroenterology and hepatology, while it appeared to affect cardiology less, since the number of citable documents in cardiology increased and

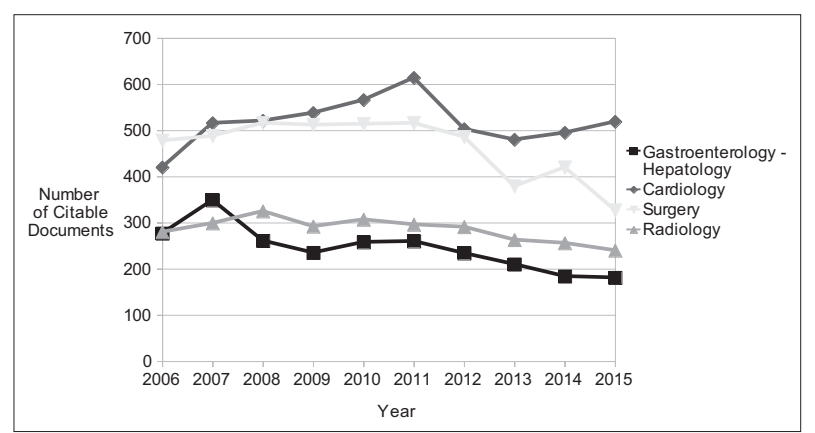

Figure 2 SCImago numbers of citable documents in the fields of gastroenterology-hepatology, cardiology, surgery and radiology in Greece during the decade 2006-2015

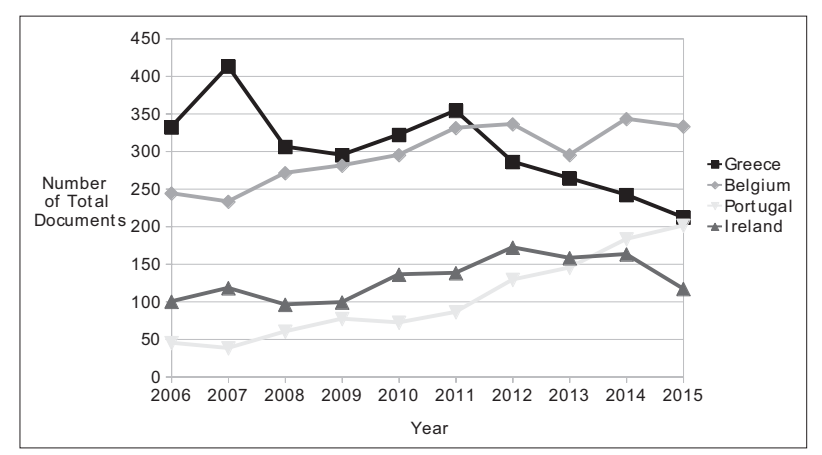

Figure 3 SCImago numbers of total documents in the fields of gastroenterology-hepatology during the decade 2006-2015: comparison of 4 European countries

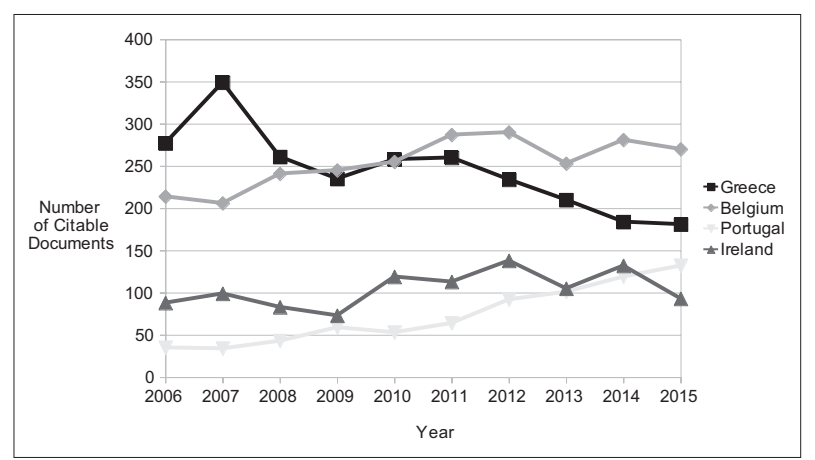

Figure 4 SCImago numbers of citable documents in the fields of gastroenterology-hepatology during the decade 2006-2015: comparison of 4 European countries virtually reached the pre-crisis levels during the last two years. It is evident from Figs. 1 and 2 that this decreasing tendency in the studied medical specialties' output started in 2011. The second major finding of our study is that the huge decline in Greek publications after 2011 rearranged the ranking of the 4 studied EU countries in terms of publication output in the field of gastroenterology and hepatology.

Both findings can, at least partially, be explained by the economic downturn that affected Greece after 2009. The carryover effect justifies the delay in the reduction of publications compared to the beginning of the economic crisis, as most of the papers published in 2009-2011 had probably been prepared in the previous years. The influence of research funding on the country's scientific output is not only obvious, but also documented. In a study published in the European Journal of Epidemiology in 2004, the authors concluded that "total research funding was significantly correlated with standardized publication rate. The correlation coefficient was $0.44(\mathrm{P}=0.05)$ indicating that nations that invested more on research had higher publication output than those that spent less on research" [6]. According to World Bank data, the research funding in Greece in 2013 represented $0.81 \%$ of the annual gross domestic product (GDP) whereas it was $2.43 \%, 1.54 \%$ and $1.33 \%$ of GDP in Belgium, Ireland and Portugal, respectively [7]. Another possible explanation for the decrease in Greek publications is the migration trend among young Greek scientists. According to a recent survey, 300,000 Greek citizens emigrated during 2010-2015, the majority being university graduates [8]. Moreover, it should be noted that the mobility of Greek researchers is the largest in the EU (73\% against the EU average of 56\% [9]). As a result, the huge "brain drain" phenomenon that is taking place in Greece due to austerity, has most likely contributed to the downward trend of Greek publications [10].

While economic recession and "brain drain" may provide some explanation for our study findings, there are still unanswered questions. First, why has the scientific productivity in cardiology recovered, while the gastroenterology and hepatology output is still diminishing? Our study cannot provide a valid explanation for this discrepancy, although scientific societies in fields such as cardiology may have more efficient dynamics to evolve towards scientific recovery. Secondly, the economic environment cannot explain the huge AAGR of Portugal in terms of both total and citable documents. One might speculate that austerity in Portugal was not as intense as it was in the case of Greece. However, the fact that the scientific output in the field of gastroenterology and hepatology in Belgium (a country that has not undergone austerity measures) and in Ireland (a country also affected by the financial burden) remained unchanged after the emergence of the crisis adds more confusion to the discrepant results of Greece and Portugal in this scientific field.

We may therefore conclude that factors unrelated to the economic environment may also have contributed to the downward trend of the Greek publication output in the scientific field of gastroenterology and hepatology. Regrettably, our study design does not permit the identification of these factors. 
Our study has 4 major strengths: 1) we obtained our data from a valid, well-accepted database; 2 ) we chose two simple measures of publication output, namely total documents and citable documents; 3) we evaluated the temporal relationship between the emergence of the economic crisis in Europe and the study outcomes; and 4) we had two comparators: the output in 3 other scientific fields within Greece, and the output in gastroenterology and hepatology of $3 \mathrm{EU}$ countries, two with a population size similar to that of Greece, and two that have experienced similar financial pressures.

Our study also has several limitations. While SCImago has numerous advantages, as mentioned above, it also has some notable disadvantages: SCImago results cannot be checked and reproduced because they are presented cumulatively and the underlying raw data are not publicly available [11]. Furthermore, the evaluation of publications was only quantitative, not taking into account their citations and the country's $\mathrm{H}$-index. The former are presented by SCImago in a way that does not allow the assessment of changes over time, and the latter is presented cumulatively at the end of the observation period only, making comparisons impossible. On the other hand, we used SCImago's citable documents as a crude measure of high quality publications. Nevertheless, the aim of the study was the assessment of publication dynamics over time. Therefore, we used AAGR to compare the publishing tendencies of the 4 specialties and the 4 countries under examination. The use of AAGR gave us the opportunity to compare the outputs of different countries without standardizing the publication numbers to the population, even though 3 of the 4 countries analyzed have similar populations. The disadvantage of AARG is that it simply calculates the average of a series of growth rates, which can lead to an inaccurate measurement of growth rates in some cases. Choosing the comparators arbitrarily is also a study limitation: surgery and radiology are closely linked to gastroenterology and hepatology in clinical practice and in research, while cardiology is the leading internal medicine specialty worldwide in terms of scientific publications and has an "interventional component" similar to that of gastroenterology. Our European comparators gave us the opportunity to compare the Greek performance in the field of gastroenterology and hepatology with that of countries of similar population, with and without fiscal austerity measures imposed on their economy. Finally, the unavailability of the number or researchers-specialists and residents in medical specialties, which could potentially play a significant role in scientific output, might also be considered as a study limitation. However, there is no reason to assume that one specialty is more affected than others by the "brain drain" phenomenon.

In conclusion, all examined specialties in Greece have been affected by the financial crisis, with a reduction in their published papers; however, in the field of gastroenterology and hepatology the effect seems more intense, whereas cardiology shows signs of recovery. Compared with other EU countries that have experienced similar financial difficulties, gastroenterology and hepatology in Greece has the biggest reduction in publications, indicating that other factors apart from research funding may possibly affect a country's research output in the respective scientific field.

\section{Summary Box}

\section{What is already known:}

- There is previous evidence that austerity measures reduce the scientific output of the affected region

- The effects of austerity on Greek medical specialties were unclear and undocumented

\section{What the new findings are:}

- Publication numbers in the scientific fields of gastroenterology-hepatology, cardiology, surgery and radiology in Greece declined after the outbreak of financial crisis, but gastroenterology and hepatology was more profoundly affected

- In comparison with Belgium, Portugal and Ireland, Greek publications in the field of gastroenterology and hepatology are the only ones to have decreased remarkably during the last five years

- The economic crisis and the "brain drain" phenomenon that has been observed in Greece during recent years are important contributing factors, albeit not the only ones

\section{References}

1. Fuyuno I. What price will science pay for austerity? Nature 2017;543:S10-S15.

2. Austerity bites. Nature 2015;523:255-256.

3. SCImago. SJR - SCImago Journal \& Country Rank [Internet]. Available from: http://www.scimagojr.com [Accessed September 3, 2016 ]

4. Falagas ME, Kouranos VD, Arencibia-Jorge R, Karageorgopoulos DE. Comparison of SCImago journal rank indicator with journal impact factor. FASEB J 2008;22:2623-2628.

5. United Nations, Department of Economic and Social Affairs, Population Division. World Population Prospects: The 2015 Revision, World Population 2015 Wallchart. ST/ESA/SER.A/378.

6. Man JP, Weinkauf JG, Tsang M, Sin DD. Why do some countries publish more than others? An international comparison of research funding, English proficiency and publication output in highly ranked general medical journals. Eur J Epidemiol 2004;19:811-817.

7. The World Bank, World Development Indicators. Research and development expenditure (\% of GDP) [Internet]. Available from: http://data.worldbank.org/indicator/GB.XPD.RSDV.GD.ZS [Accessed April 7, 2017]

8. Labrianidis L, Pratsinakis M. Greece's new emigration at times of crisis. Hellenic Observatory LSE 2016;99:5-20.

9. Theodoropoulos D, Kyridis A, Zagkos C, Konstantinidou Z. "Brain drain" phenomenon in Greece: young Greek scientists on their way to immigration, in an era of "crisis". Attitudes, opinions and beliefs towards the prospect of migration. J Educ Hum Dev 2014;3:229-248.

10. Trachana V. Austerity-led brain drain is killing Greek science. Nature 2013;496:271.

11. Mañana-Rodríguez J. A critical review of SCImago Journal \& Country Rank. Res Eval 2015;24:343-354. 